\title{
ProBNP processing is decreased by obesity in patients with heart failure
}

\author{
Lynley K. Lewis ${ }^{1}$, Sara D. Raudsepp ${ }^{1}$, Tim C. R. Prickett ${ }^{1}$, Tim G. Yandle ${ }^{1}$, Chris J. Pemberton ${ }^{1}$, \\ A. Mark Richards ${ }^{1,2}$
}

${ }^{1}$ Christchurch Heart Institute, Department of Medicine, University of Otago, Christchurch, New Zealand; ${ }^{2}$ Cardiac Department, Cardiovascular Research Institute, National University Heart Centre, National University of Singapore, Singapore, Singapore

Correspondence to: Lynley K. Lewis. Christchurch Heart Institute, Department of Medicine, University of Otago, 2 Riccarton Avenue, Christchurch 8011, New Zealand Email: lynley.lewis@otago.ac.nz.

Provenance: This is an invited article commissioned by the Guest Section Editor Dr. Ying Zhao (Department of Laboratory Medicine, the First Affiliated Hospital, College of Medicine, Zhejiang University, Hangzhou, China).

Response to: Nishikimi T, Nakagawa Y. Does impaired processing of pro-B-type (or brain) natriuretic peptide cause decreased plasma BNP levels in obese heart failure patients? Ann Transl Med 2019;7:S221.

Parcha V, Arora P. Glycosylation of natriuretic peptides in obese heart failure: mechanistic insights. Ann Transl Med 2019;7:611.

Submitted Nov 27, 2019. Accepted for publication Dec 09, 2019.

doi: $10.21037 /$ atm.2019.12.81

View this article at: http://dx.doi.org/10.21037/atm.2019.12.81

We thank Doctors Parcha and Arora (1), and Nishikimi and Nakagawa (2) for their interest in our study reporting that proBNP not glycosylated at threonine 71 (T71) was decreased in obese patients with chronic heart failure (3). We appreciate these authors taking the time to provide well-constructed overviews of the current literature relating to processing of proBNP and the known links between NT-proBNP and BNP and obesity. While BNP and NTproBNP measurement is mandated in the diagnosis and risk stratification of heart failure (4), levels of these peptides are decreased with obesity, complicating their use in the clinical assessment of heart failure in obese individuals (5). The reasons for this decrease have not been adequately explained although many theories have been debated $(2,3)$.

Our study investigated the relationship between proBNP glycosylation and obesity in patients with heart failure. Glycosylation of proBNP at T71 prevents cleavage to form bioactive BNP1-32 and its congener NT-proBNP $(6,7)$. As accurate measurement of peptide glycosylation is difficult to assess, we chose to measure T71 that was not glycosylated. Using antibodies that only detect non-glycosylated proBNP sites, we developed three immunoassays, all of which had a common antibody directed towards the C-terminal end of proBNP. Assay specificity was conferred by coupling this antibody with different amino terminal proBNP antibodies resulting in assays that detected either total proBNP, proBNP not glycosylated at threonine 71 or proBNP not glycosylated centrally. Using these assays we showed that the rate of proBNP glycosylation was much higher centrally than at T71, implying differential regulation of glycosylation at these two sites in our patients with heart failure. We also found that the level of total proBNP was not different between heart failure patients with BMI above or below $30 \mathrm{~kg} / \mathrm{m}^{2}$. In contrast the plasma concentrations of proBNP not glycosylated at T71 were lower in patients with BMI $>30 \mathrm{~kg} / \mathrm{m}^{2}$, presumably due to increased glycosylation of T71. Increased glycosylation at T71 should result in lower levels of NT-proBNP and BNP in this group, due to decreased proBNP processing, and this was confirmed by lower plasma concentrations of NT-proBNP in our higher BMI heart failure sub-cohort. Further confirmation was provided by the proBNP:NTproBNP and proBNP:nonglycosylated T71 ratios which were higher in those cases with BMI $>30 \mathrm{~kg} / \mathrm{m}^{2}$ group. Together, these findings are consistent with increased glycosylation at T71 underlying at least part of the reduced plasma concentrations of BNP and NT-proBNP observed in obesity.

We used the commercially available Roche NT-proBNP 2-site chemiluminescent immunoassay to measure NTproBNP in this study as this assay is widely used in heart 
failure diagnosis and prognosis worldwide. NT-proBNP and $\mathrm{BNP}$ are processed from proBNP in equimolar amounts, and plasma concentrations of both are employed to assist in the diagnosis of heart failure, with the longer half-life of NT-proBNP making it more suitable for routine measurement. Additionally, measurement of plasma BNP in patients with heart failure is complicated as circulating levels of proBNP are at least twice that of BNP in heart failure (8) and all available BNP assays also measure proBNP, thus overestimating the actual BNP concentration. We have developed an assay specific for BNP1-32 that does not cross-react with proBNP or BNP metabolites (9) and studies assessing both overall BNP immunoreactivity and specific BNP1-32 itself in this patient cohort are currently underway in our laboratory.

Combined angiotensin II receptor blocker/neprilysin inhibitor (ARNI) constitutes a clear advance in antiheart failure pharmacotherapy which is becoming widely prescribed. Nougue et al. reported that the ARNI sacubitril/ valsartan increased T71 glycosylation (10), implying that ARNI treatment resulted in decreased proBNP processing. However, the measurement of proBNP processing in that study was complex and involved calculating T71 glycosylation by mass spectrometry following enzymatic deglycosylation and tryptic digestion of the sample and then determination of the proBNP processing rate at each time point in their study from the following equation: [BNP $=\mathrm{f}(\mathrm{NT}-$ proBNP $) /$ median T71 proBNP glycosylation]. As proposed by Parcha and Arora (1), analysing samples from patients treated with ARNI with our assays coupled to an NTproBNP assay should enable the assessment of T71 glycosylation in a more direct manner.

Increased expression of GalNAc-transferases have been shown to result in increased glycosylation and secretion of proBNP in patients with heart failure (2). When combined with our results demonstrating that rates of proBNP not glycosylated at $\mathrm{T} 71$ are different between obese and normal weight patients with heart failure, these studies emphasise that more research is needed to elucidate the mechanisms regulating proBNP processing in heart failure. Whilst we cannot discount that the post-translational modification at T71 occurs via an alternative non-glycosylation pathway, we specifically chose our antibodies because their epitopes target known proBNP glycosylation sites and, given our results, believe glycosylation is relevant to reduced plasma concentrations of BNP/NT-proBNP in obesity.

The concentrations of circulating BNP and NTproBNP are potentially influenced by many factors other than proBNP glycosylation, including concentrations and activity of the proBNP processing enzymes furin and corin, concentrations of the active and clearance receptors (NPRA and NPRC), activity of proteolytic enzymes including neprilysin and insulin degrading enzyme, and metabolic status and renal function. Using our assays we can determine lack of glycosylation at specific proBNP sites, and despite the complexities of BNP biochemistry, we were able to show differences in T71 glycosylation between obese and non-obese patients with heart failure, providing a new mechanism which may explain in part the decreased levels of BNP and NT-proBNP observed in obese individuals.

We acknowledge that our study included a limited number of patients from a single centre and leaves many questions unanswered. We appreciate the comments from both Parcha and Arora (1), and Nishikimi and Nakagawa (2) regarding our findings. Quantifying concentrations of specific proBNP glycosylation forms in sub-groups of patients with heart failure, and in particular assessing proBNP and BNP forms in patients treated with ARNIs will expand knowledge of the mechanisms underlying proBNP processing and will increase understanding of the associations between BNP production, $\mathrm{BMI}$ and heart failure pathogenesis. We look forward to using our assays to further advance this field.

\section{Acknowledgments}

Funding for this project was provided by The Heart Foundation of New Zealand and the Health Research Council of New Zealand.

\section{Footnote}

Conflicts of Interest: The authors have no conflicts of interest to declare.

Ethical Statement: The authors are accountable for all aspects of the work in ensuring that questions related to the accuracy or integrity of any part of the work are appropriately investigated and resolved.

\section{References}

1. Parcha V, Arora P. Glycosylation of natriuretic peptides in obese heart failure: mechanistic insights. Ann Transl Med 2019;7:611.

2. Nishikimi T, Nakagawa Y. Does impaired processing of 
pro-B-type (or brain) natriuretic peptide cause decreased plasma BNP levels in obese heart failure patients? Ann Transl Med 2019;7:S221.

3. Lewis LK, Raudsepp SD, Prickett TCR, et al. ProBNP That Is Not Glycosylated at Threonine 71 Is Decreased with Obesity in Patients with Heart Failure. Clin Chem 2019;65:1115-24.

4. Yancy CW, Jessup M, Bozkurt B, et al. 2017 ACC/ AHA/HFSA Focused Update of the 2013 ACCF/AHA Guideline for the Management of Heart Failure: A Report of the American College of Cardiology/American Heart Association Task Force on Clinical Practice Guidelines and the Heart Failure Society of America. Circulation. 2017;136:e137-61.

5. Bayes-Genis A, Lloyd-Jones DM, van Kimmenade RR, et al. Effect of body mass index on diagnostic and prognostic usefulness of amino-terminal pro-brain natriuretic peptide in patients with acute dyspnea. Arch Intern Med 2007;167:400-7.

Cite this article as: Lewis LK, Raudsepp SD, Prickett TCR, Yandle TG, Pemberton CJ, Richards AM. ProBNP processing is decreased by obesity in patients with heart failure. Ann Transl Med 2020;8(4):135. doi: 10.21037/atm.2019.12.81
6. Semenov AG, Postnikov AB, Tamm NN, et al. Processing of pro-brain natriuretic peptide is suppressed by O-glycosylation in the region close to the cleavage site. Clin Chem 2009;55:489-98.

7. Nishikimi T, Nakagawa Y, Minamino N, et al. Pro-Btype natriuretic peptide is cleaved intracellularly: impact of distance between O-glycosylation and cleavage sites. Am J Physiol Regul Integr Comp Physiol 2015;309:R639-49.

8. Yandle TG, Richards AM, Gilbert A, et al. Assay of brain natriuretic peptide (BNP) in human plasma: evidence for high molecular weight $\mathrm{BNP}$ as a major plasma component in heart failure. J Clin Endocrinol Metab 1993;76:832-8.

9. Lewis LK, Raudsepp SD, Yandle TG, et al. Development of a BNP1-32 Immunoassay That Does Not Cross-React with proBNP. Clin Chem 2017;63:1110-7.

10. Nougué H, Pezel T, Picard F, et al. Effects of sacubitril/ valsartan on neprilysin targets and the metabolism of natriuretic peptides in chronic heart failure: a mechanistic clinical study. Eur J Heart Fail 2019;21:598-605. 